\title{
PREDICTION MODELS OF SERVICE PERFORMANCE DEGRADATION FOR METRO SHIELD TUNNELS
}

\author{
Junhua Xiao ${ }^{1 *}$, Dong Liang ${ }^{1}$, Xinzhong Nong ${ }^{2},{\text { Nan } W u^{3} \text { and Jinrong Song }}^{1}$ \\ ${ }^{1}$ Key Laboratory of Road and Traffic Engineering of the Ministry of Education, Tongji University, Shanghai, China \\ ${ }^{2}$ Guangzhou Metro Design \& Research Institute Co., Ltd., Guangzhou, China \\ ${ }^{3}$ Beijing Jiaotong University, Beijing, China \\ * Corresponding author
}

\begin{abstract}
In this study, two models were proposed for predicting the rapid and long-term degradations in the serviceability (indicated by the tunnel serviceability index) of metro shield tunnels in soft ground. Both models were validated by applying them to a metro shield tunnel in soft ground in a city in southern China against historic monitoring data. It was found that the rapid serviceability degradation of shield tunnels can be categorized into two distinct modes, slowing-down degradation and recovery-and-slowing-down degradation. Our Sigmoid function-based prediction model can well predict both modes of rapid degradation. The long-term serviceability degradation of shield tunnels can be categorized into three distinct modes, concave-curve, S-shaped-curve, and convex-curve modes. Our inverse exponential function-based prediction model can well predict all three modes of long-term degradation. In a case study, both models demonstrated good prediction capabilities in their respective application scopes.
\end{abstract}

\section{Introduction}

Metro shield tunnels in China are increasingly experiencing defects, despite most of them being put into operation less than 10 years ago. Metro shield tunnels in soft ground (for example, some tunnels in Shanghai and Nanjing) are particularly prone to defects such as differential settlement, lateral convergence, water seepage and leakage, cracking in the lining surface, spalling of the lining, degradation of concrete, and erosion of steel bars (Yuan, et al., 2012). This makes tunnel monitoring and maintenance more challenging for metro operators. Thus, it is important to predict the service performance of metro shield tunnels in soft ground.

Most of the methods currently available for predicting the degradation of shield tunnel service performance are based on a single quantitative parameter. Such prediction models can be categorized into two distinct types, probabilistic and deterministic models. Probabilistic models take into account randomness in tunnel structure and peripheral environment and are advantageous for solving uncertain problems. However, the introduction of the randomness may result in a certain degree of randomness in the variation of structural performance. Yuan et al. (2013) prioritized possible tunnel defects, by employing the failure mode and effect analysis (FMEA) approach to calculate their risk priority numbers (RPN), and predicted the accumulative damage of each defect by employing a Weibull probabilistic model and a lognormal probabilistic model (both of which were based on historic data), thereby realizing a better assessment of the systematic risks of shield tunnels. To cope with increasing deformation of shield tunnels in Shanghai soft clay, Huang et al. (2017) performed a historic data-based probabilistic assessment of the long-term and short-term evolutions of the deformational performance of shield tunnels, by using the horizontal convergence in the inner radius as the indicator of tunnel performance (which was assumed to be of parabolic transformation). A deterministic prediction model may be a mechanical method, an empirical method, or a combination of mechanical and empirical methods. Liu et al. (2017) developed a model for analysing the erosion and degradation of the tunnel lining under the coupled effects of external hydraulic pressure and eroding ions. Wei et al. (2009) predicted the long-term settlement of the ground above the axis of the shield tunnel, using eight common mathematical models, and compared the fitting accuracies of the models.

All the above prediction methods are based on a single parameter and are insufficient to describe the overall performance of shield tunnels. With improved systems available for assessing the overall performance of shield tunnels, more objective and accurate methods have been developed for computing assessment indicators. Based on six common types of distress, namely, relative settlement, differential settlement, convergence deformation, water leakage, cracking and spalling, Li et al. (2017) propose a comprehensive tunnel serviceability index (TSI) by using an expert scoring method, which is used to evaluate the overall condition of a shield tunnel in soft ground. Compared with other assessment indicators, TSI is more systematic, accurate, and quantifiable.

In this study, two models were developed for predicting typical modes of long-term and short-term degradations in the service performance of tunnels in soft ground, using TSI as the service performance indicator. The applicability and the methods of application of both models were analysed. Both models were 
verified against historic monitoring data of a metro shield tunnel in soft ground in a city in southern China.

\section{Model for predicting rapid degradation}

\subsection{Modes of rapid degradation of shield tunnels}

The serviceability of shield tunnels in soft ground may degrade significantly and rapidly during the initial stage of operation, owing to poor geological conditions. Tunnel operators need to take timely maintenance, such that defects are remedied to recover tunnel serviceability and service conditions are improved to mitigate subsequent degradations. During the stage of rapid degradation, the serviceability degradation of shield tunnels may exhibit two distinct modes, depending on the maintenance characteristics of tunnels, as shown in Fig. 1.

Fig. 1 Modes of serviceability degradation of shield tunnels during the stage of rapid degradation

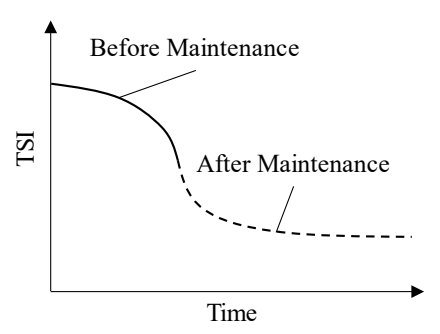

(a)

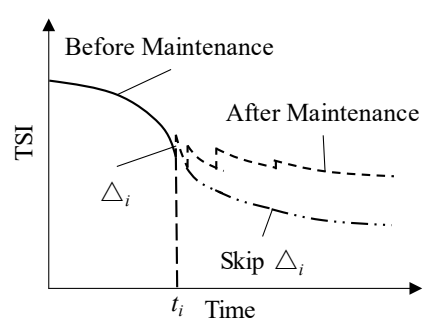

(b)
Slowing-down mode: The degradation of the tunnel slows down after maintenance and approximates a stable level, exhibiting an S-shaped pattern, as shown in Fig. 1(a).

Recovery-and-slowing-down mode: The performance of tunnel recovers after maintenance and subsequently degrades at decreasing rates, approximating a stable level. The maintenance of this kind is generally not completed at one time. The serviceability degradation in this mode exhibits a similar S-shaped pattern to the slowing-down mode of degradation, if the recovered $T S I(\Delta i)$ after each instance of maintenance $\left(t_{i}\right)$ is neglected, as shown in Fig. 1(b).

\subsection{Prediction model based on sigmoid curve}

The slowing-down mode of serviceability degradation can be expressed as the following Sigmoid function:

$T S I=A-\frac{a}{1+e^{-k\left(x-x_{C}\right)}}$

While the recovery-and-slowing-down mode of serviceability degradation can be expressed as:

$T S I=\left\{\begin{array}{lr}A-\frac{a}{1+e^{-k\left(x-x_{c}\right)}}, & x<t_{1} \\ A-\frac{a}{1+e^{-k\left(x-x_{c}\right)}}+\sum_{1}^{i} \triangle_{i}, & t_{i} \leq x<t_{i+1} \\ A-\frac{a}{1+e^{-k\left(x-x_{c}\right)}}+\sum_{1}^{m} \triangle_{i}, & t_{m} \leq x\end{array}\right.$

Where TSI is the predicted tunnel serviceability, with a value between 4 and 5 categorized as Very Good, a value between 3 and 4 categorized as Good, a value between 2 and 3 categorized as Fair, a value between 1 and 2 categorized as Poor, and a value between 0 and 1 categorized as Very Poor (Li et al. 2017), $A$ is the initial tunnel serviceability during the period of rapid degradation, $x$ is the service life, $a, k$, and $x_{c}$ are regression coefficients, and $m$ is the total instances of maintenance for the recovery-and-slowing-down mode of degradation.

Fig. 2 presents the serviceability degradation curves generated by Equation (1), at a fixed value of 5.0 for $A$ and different values for each of the regression coefficients. Regression coefficient $a$ represents the effect of maintenance and affects the value of TSI after serviceability degradation stabilizes, with a bigger value of $a$ resulting in a value of TSI closer to 0 . Regression coefficient $x_{c}$ represents the time point when the effect of maintenance begins to manifest itself and affects the duration of the period of rapid degradation. The slope of the degradation curve peaks at $k / 4$ when $x_{c}=x$, and decreases and approximates 0 when $x_{c}$ further deviates from the value of $x$. Regression coefficient $k$ represents the rate of degradation.

Figure 2 Parameter analysis of the prediction model for the rapid decay period

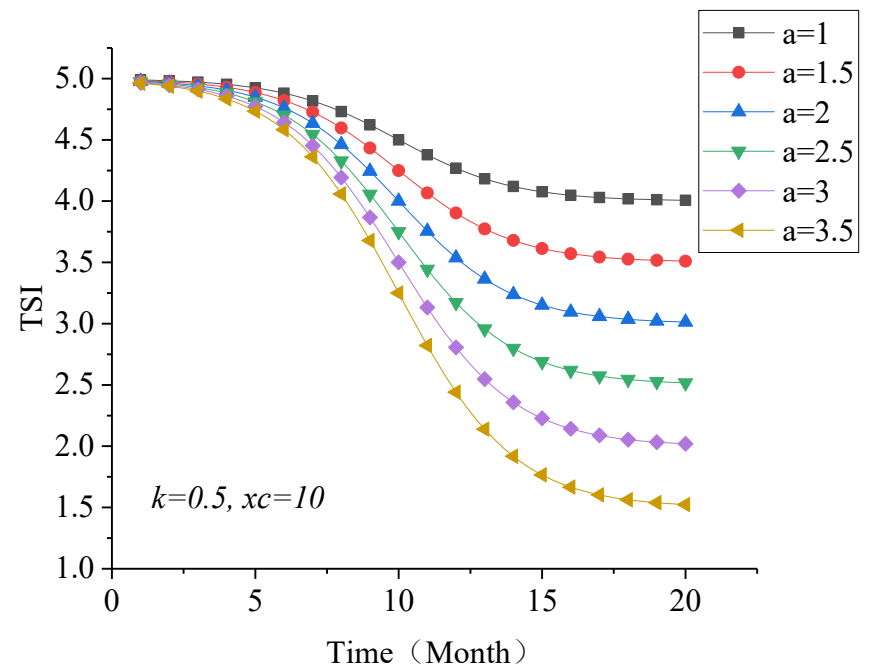

(a)

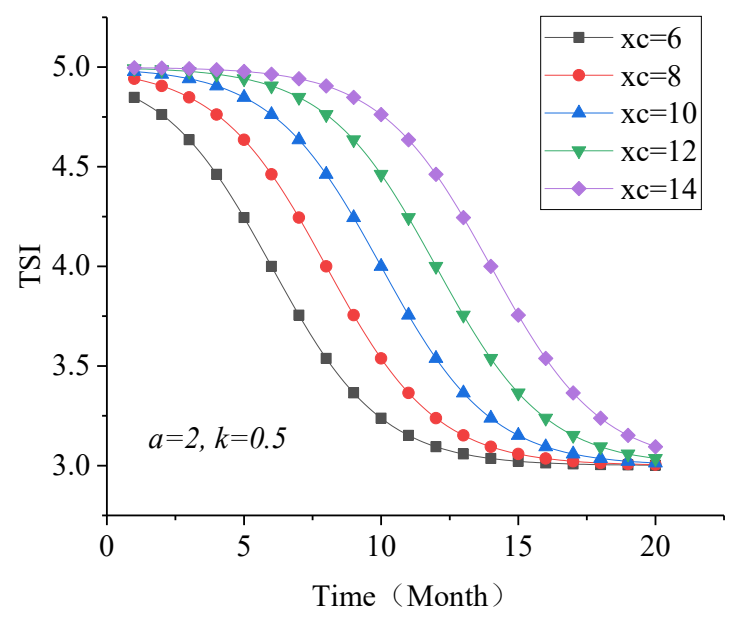

(b) 


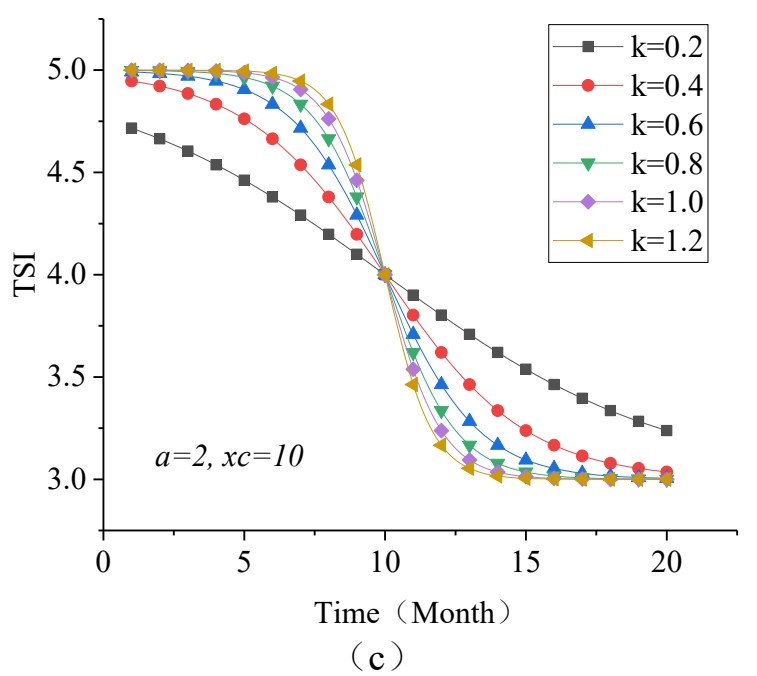

2.3 Use of the prediction model for the rapid decay period

Step 1: The model is applicable to the initial stage of operation when the serviceability of the shield tunnel rapidly degrades and then gradually stabilizes, but is not applicable to any scenario where a tunnel undergoes slow serviceability degradation and requires long cycles of maintenance. In this paper, the stage of rapid degradation is defined as a period in the first five years of operation when the TSI of a tunnel continuously decreases at rates higher than $0.05 /$ quarter $(0.2 /$ year) for more than a year, and it ends at the moment when TSI gradually decreases at rates lower than 0.05 /quarter (0.2/year).

Step 2: Calculate the initial TSI $(A)$ of the tunnel, using the data obtained from the first monitoring of the tunnel.

Step 3: Determine the control value of TSI $(B)$ - target TSI determined according to the limit value of the major distress for the tunnel. For example, the bolt begins to yield when the convergence reaches $7.7 \% 0 \mathrm{D}(\mathrm{D}$ is the designed external diameter) (Wang and Zhang, 2013) — for the end value of the stage of rapid degradation, and add points $(+\infty, \mathrm{B})$ into the historic monitoring data of the tunnel for curve fitting. Thereby, the final prediction curve can be obtained. According to the mathematical characteristics of the model, the validity period is from 0 to $2 x_{c}$. When the model is used for predicting longterm degradation, the prediction curve will approach the $B$ value indefinitely, which goes against the realistic law of tunnel service performance decaying with time.

\section{Model for predicting long-term degradation}

\subsection{Modes of long-term degradation of shield tunnels}

The stage of rapid degradation is followed by long-term degradation. Based on engineering experience and reasonable assumptions, shield tunnels in soft ground in China exhibit three typical modes of long-term degradation, as shown in Fig. 3.
Figure 3 Typical degradation modes for long-term decay periods

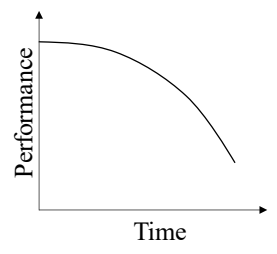

(a)

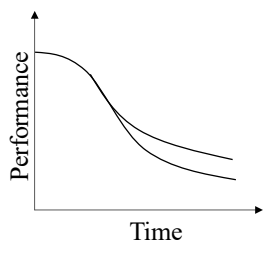

(b)

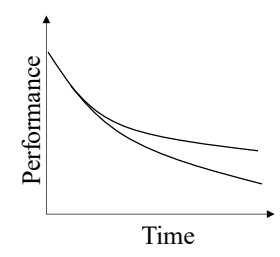

(c)
Convex curve: The serviceability of the tunnel degrades first at lower rates and then at higher rates, as shown in Fig. 3(a). Initially, the tunnel is adequately capable of resisting various defects and, thus, experiences lower rates of degradation. After various minor defects have emerged, the serviceability of the tunnel gradually degrades. When the serviceability degradation reaches a certain degree and tunnel maintenance fails to resist the trend of degradation, the serviceability of the tunnel degrades rapidly.

S-shaped curve: The serviceability degradation of the tunnel exhibits an S-shaped pattern, as shown in Fig. 3(b). Compared with the convex curve in Fig. 3(a), the S-shaped curve exhibits a similar trend of variation in the early stage. During this stage, the serviceability of the tunnel can be stabilized, if the tunnel is appropriately maintained by the operator.

Concave curve: The serviceability of the tunnel degrades first at higher rates and then at lower rates, as shown in Fig. 3(c). This pattern usually occurs with tunnels in soft ground and may occur with inappropriately constructed tunnels. The variation trend of the curve mainly depends on the maintenance program and its timely implementation by the tunnel operator.

\subsection{Prediction model based on an inverse exponential function}

The long-term serviceability degradation of shield tunnels can be predicted using the following equation, which is a simple but good representation of the three typical modes of structural performance degradation of tunnels:

$T S I=\operatorname{TSI}_{0}\left[1-e^{-\left(\frac{\alpha}{x}\right)^{\beta}}\right]$

Where $T S I$ is the indicator of tunnel serviceability, $T S I_{0}$ is the initial tunnel serviceability of the long-term serviceability degradation of the tunnel, $x$ is the service life, and $\alpha$ and $\beta$ are regression coefficients.

Equation (3) reveals that, when $\alpha \geq 0$ and $\beta \geq 0$, TSI monotonically decreases from $T S I_{0}$ to 0 . The serviceability degradation curve varies with regression coefficients $\alpha$ and $\beta$. Fig. 2 presents curves of the three typical modes of serviceability degradation at a fixed $T_{S I_{0}}$ value of 5.0 and various values of $\alpha$ and $\beta$.

Increase in the value of $\beta$ results in the curve changing from a concave shape to an S shape, as shown in Fig. 4(a); increase in the value of $\alpha$ results in the curve changing from a concave shape to an $\mathrm{S}$ shape and then to a convex shape. Furthermore, 
with the value of $\alpha$ fixed, the curves with different values of $\beta$ all pass through the point $\left(\alpha, 63.2 \% T S I_{0}\right)$. This indicates that $\alpha$ represents the service life of the tunnel when the serviceability of the tunnel degrades to $63.2 \%$ of the initial serviceability and, thus, can be taken as the life parameter of the prediction model.

Figure 4 Parameter analysis of the long-term prediction model

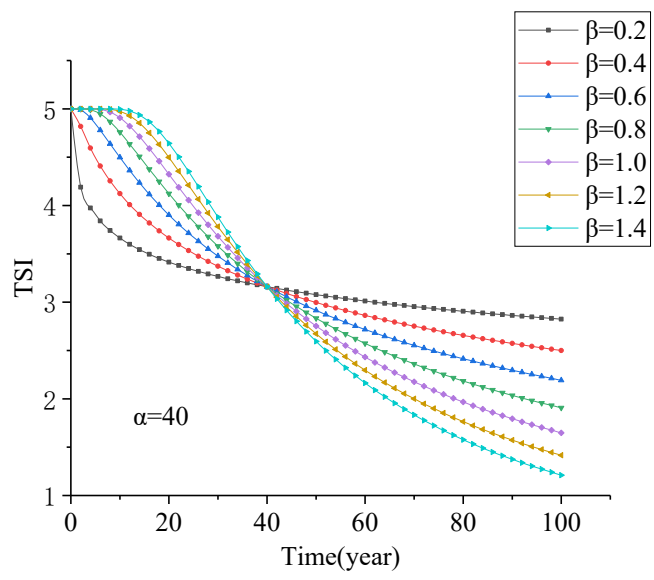

(a)

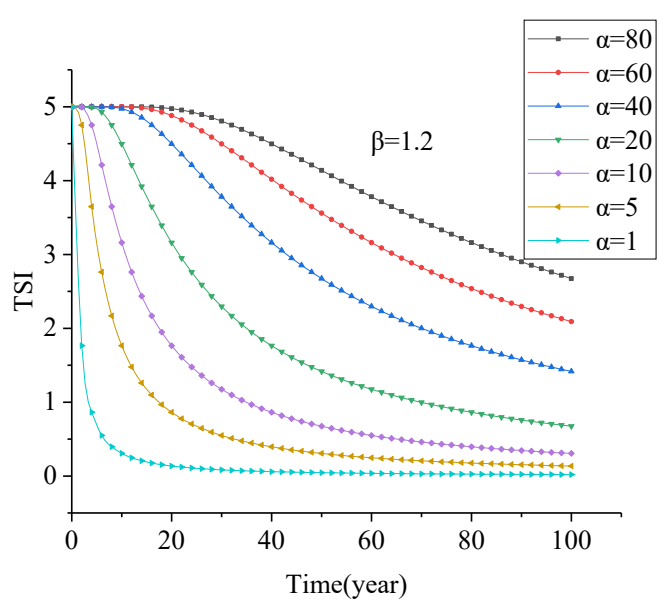

(b)

\subsection{Use of long-term prediction model}

Figure 5 Functions of the long-term prediction model

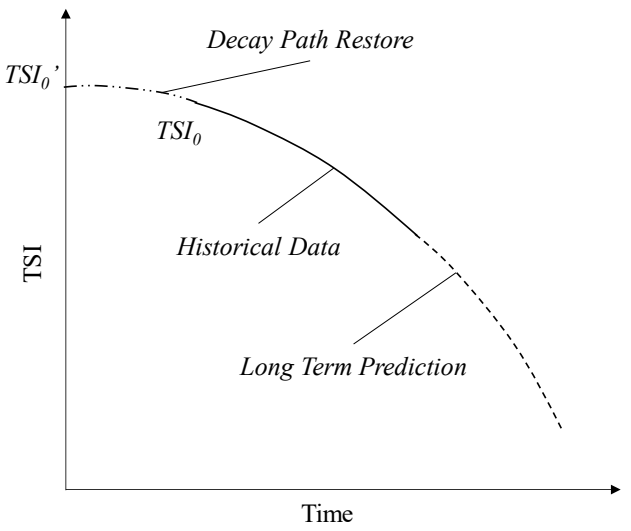

The model described in Equation (3) is not only capable of predicting the remaining life and serviceability of the longterm degradation of tunnels but also reconstructing the degradation paths during a period without observation data. The model was established in the following steps:

Step 1: Determine the initial serviceability of tunnel TSI 0 . If a tunnel exhibits rapid serviceability degradation during the initial stage of its operation, the end of this stage of rapid degradation is taken as the starting point of the long-term degradation and the TSI at this time point is taken as $T S I_{0}$; if a tunnel does not exhibit such a stage of rapid degradation, then the time point when the first tunnel monitoring is performed is taken as the starting point of the long-term degradation, and the TSI calculated using the data obtained from the first monitoring is taken as $T S I_{0}$.

Step 2: Input all the historic data into Equation (3) for fitting, and update the prediction model by continuously inputting new data obtained into the equation. Thereby, the long-term serviceability degradation of the tunnel can be predicted.

Step 3: To reconstruct the degradation path of a tunnel during a period without monitoring data, assume a point of $\left(0, T S I_{0}{ }^{\prime}\right)$ and a value of $T S I_{0}{ }^{\prime}$ below 5.0, perform a regression analysis using existing data, and select the curve with the best fit as the degradation path.

\section{Case study}

The prediction models were applied to a metro shield tunnel in a city in southern China. The total length of the tunnel is 0.97 $\mathrm{km}$. The external diameter of the tunnel is $6.2 \mathrm{~m}$, and the internal diameter is $5.5 \mathrm{~m}$. The ring width and thickness are 1.2 $\mathrm{m}$ and $0.35 \mathrm{~m}$, respectively. The lining ring consists of six segments, specifically, one key segment, two adjacent segments, and three standard segments, and the segments are assembled with 12 bending bolts (nominal diameter is $30 \mathrm{~mm}$ and mechanical property grade is 5.8) and EPDM sealing gaskets. The rings are assembled with staggered joint. The tunnel was put into operation in 2010 .

This tunnel was constructed in soft soil with poor engineering properties, as shown in Fig. 6. Under the tunnel was a thick layer of muddy, silty clay, and the 2.0-m-thick layer of soil under the ground surface was rich in ground water. As a result, the tunnel experienced rapid settlements after being put into operation. In addition, the disturbance of the soil around the tunnel due to large-scale engineering activities, and the remarkable dynamic effects because of the small-radius curve of the subway line, both aggravating the development of settlement. The settlement of the tunnel induced various other structural defects such as increased transverse convergences, water seepages and leakages, and cracks in the lining surface.

The patrol inspection records and monitoring data of the tunnel operator show that the average cumulative settlement of the tunnel from its commission to the end of 2013 was $41.2 \mathrm{~mm}$, though just a small number of defects were induced; altogether nine water seepages were detected during the three-year period, and there were two cracks in lining surface detected, as shown in Fig. 7(a). In the subsequent one-year period, the tunnel increasingly experienced defects, owing to the lack of timely maintenance. In 2014, 13 water leakages and seepages were 
detected, among which six deteriorated into water dripping, and 15 cracks in the lining surface were detected, as shown in Fig. 7(b). The more frequent occurrences of defects resulted in rapid settlements of the tunnel. As of the end of 2014, the average accumulative settlement reached $76.9 \mathrm{~mm}$.

Considering that different sections of the tunnel possibly experienced different serviceability degradations, the tunnel was divided into sections of approximately $200 \mathrm{~m}$ in length. The prediction models were applied to a middle section of the tunnel, measuring $214.8 \mathrm{~m}$ in length and consisting of 179 rings (the target section), by calculating the six basic indicators for calculating the TSI using historic monitoring data and inputting the calculated values of the indicators into Equation (4) (Li et al., 2017). Table 1 presents the calculations. Figures 8(a) and Figure 6 Soil condition of the tunnel 8(b) present the actual absolute values and degradation rates of the TSI of the target section.

$$
\begin{aligned}
& T S I=5.23-0.16 \sqrt{S_{\text {ave }}}-0.01 S_{\text {diff_ave }}-0.09 c_{\text {ave }}- \\
& 0.08 d_{l}-0.05 d_{c}-0.50 d_{s}
\end{aligned}
$$

where $S_{\text {ave }}$ is the average settlement difference between the observation point with the least settlement with other observation points, $S_{\text {diff ave }}$ is the average ratio of settlement difference to horizontal distance between all adjacent observation points, $C_{\text {ave }}$ is the average convergence deformation, $d_{l}$ is the water leakage area per 100 rings, $d_{c}$ is the cracking length per 100 rings, and $d_{s}$ is the spalling area per 100 rings (Li et al., 2017).

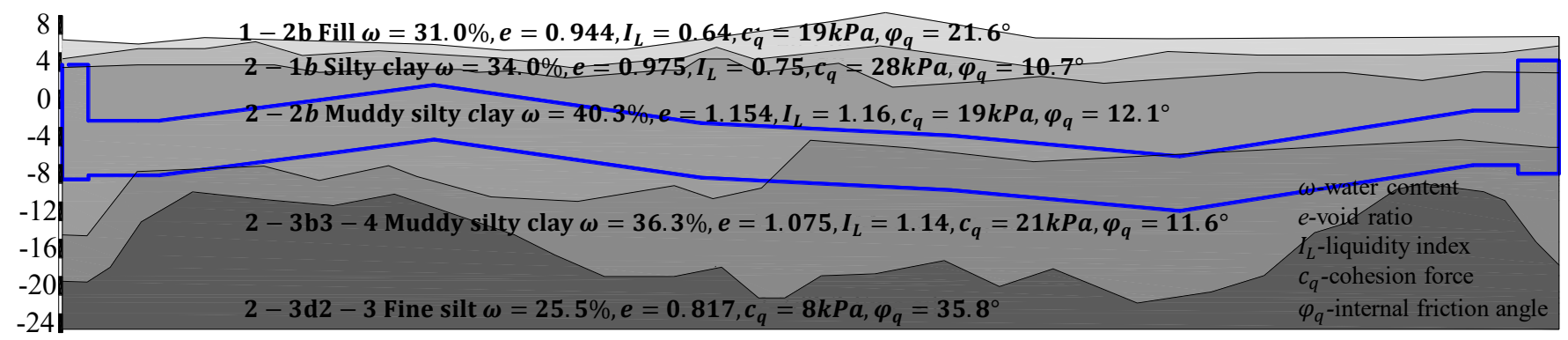

Figure 7 Rapid development of tunnel distresses

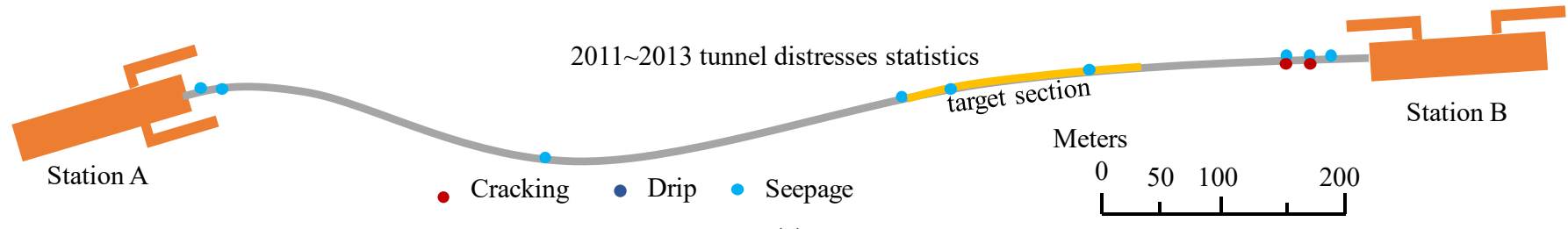

(a)

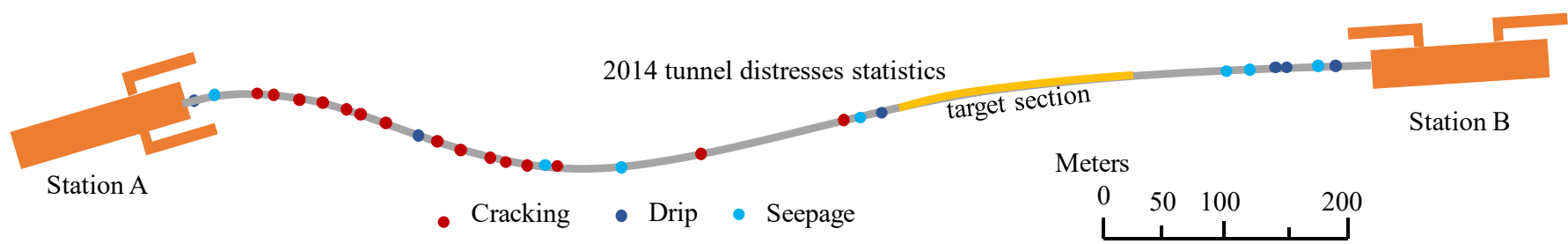

(b)

\begin{tabular}{|c|c|c|c|c|c|c|c|}
\hline Time & $\begin{array}{l}S_{\text {ave }} \\
(\mathrm{mm})\end{array}$ & $\begin{array}{l}S_{\text {diffave }} \\
(\mathrm{mm} / 100 \mathrm{~m})\end{array}$ & $\begin{array}{l}c_{\text {ave }} \\
(\% \mathrm{D})\end{array}$ & $\begin{array}{l}d_{l} \\
\left(\mathrm{~m}^{2} / 100 \text { rings }\right)\end{array}$ & $\begin{array}{l}d_{c} \\
(\mathrm{~m} / 100 \text { rings })\end{array}$ & $\begin{array}{l}d_{s} \\
\left(\mathrm{~m}^{2} / 100 \text { rings }\right)\end{array}$ & $T S I$ \\
\hline $2010 / 12$ & 11 & 7.89 & 0.94 & 0 & 0 & 0 & 4.54 \\
\hline $2014 / 10$ & 26 & 23.01 & 2.74 & 0 & 0 & 0 & 3.94 \\
\hline
\end{tabular}

Table 1 Cases of TSI calculation

As shown in Fig. 8, the serviceability degradation of the target section gradually increased after three years of operation, with TSI decrease rates during the period from December 2013 to June 2015 higher than 0.2 year. This satisfies the applicability conditions of the model for predicting rapid serviceability degradation, as described above. Assuming that at the end of 2014 the tunnel operator needed to use the rapid degradation prediction model to assist the maintenance required to resist the rapid serviceability degradation of the tunnel, the prediction model can be established as follows:

Firstly, calculate the TSI using the data obtained from the first monitoring performed in December 2010 and take the calculated TSI (4.54) as $A$ value. 
Secondly, assume a target TSI value of 3.5( $B$ value $)$ - the median value for a good level of serviceability - based on the TSI value of 3.94 at the end of 2014.

Finally, input the TSI values calculated using the data of the period from December 2010 to October 2014 into Equation (1) for fitting - as the pattern of serviceability degradation fits with the slowing-down mode of rapid degradation illustrated in Fig. 8(a). The resulting prediction of the rapid serviceability degradation of the target section of the tunnel can be expressed as Equation (5):

$T S I=4.54-\frac{1.042}{1+e^{-0.099(x-45.4)}}, \quad(\mathrm{R}=0.997)$

Figure 8 Decay process of the target section

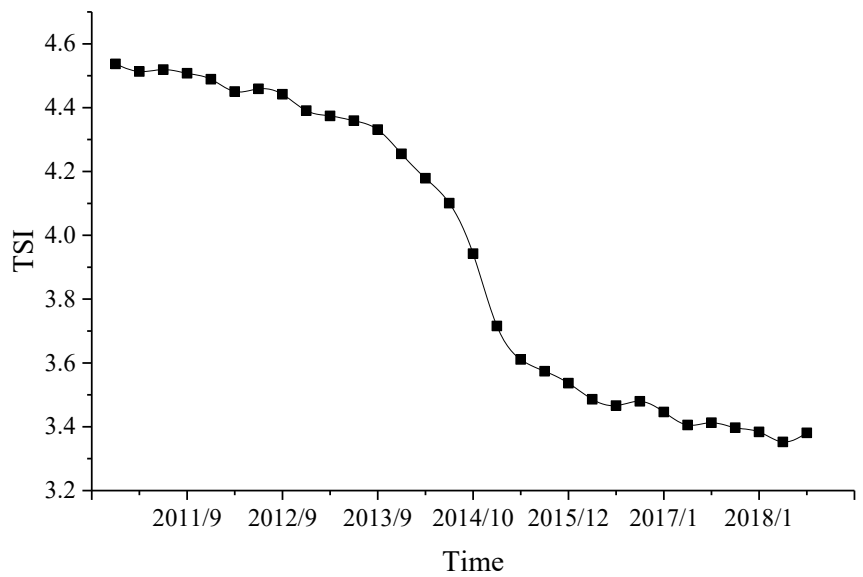

(a)

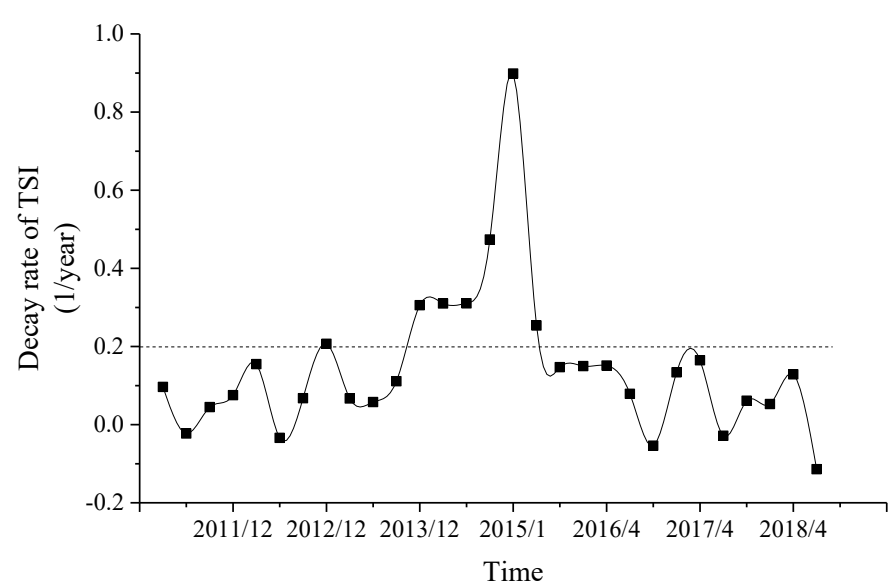

(b)

Fig. 9 presents the TSI values prior to July 2018, predicted using the model. The figure shows that there are only small disagreements between the fitting and actual TSI values for the period from December 2010 to October 2014, thereby confirming the good fitting effect of the model. In the subsequent operation of the tunnel, the operator remedied water seepages and leakages by grouting behind the shaft lining, repairing cracks in the lining surface using cement mortar, and controlling tunnel deformation by microdisturbance. Thereby, the serviceability degradation of the tunnel was slowed down, with the TSI controlled in the range of $3.38-3.61$. As the actual effect of maintenance is slightly lower than the target effect, the predicted TSI values for the period from January 2015 to July 2018 are slightly higher than the actual values. Nevertheless, the predicted and actual TSI values exhibit very similar patterns of variation.

Figure 9 Prediction curve of the rapid decay period

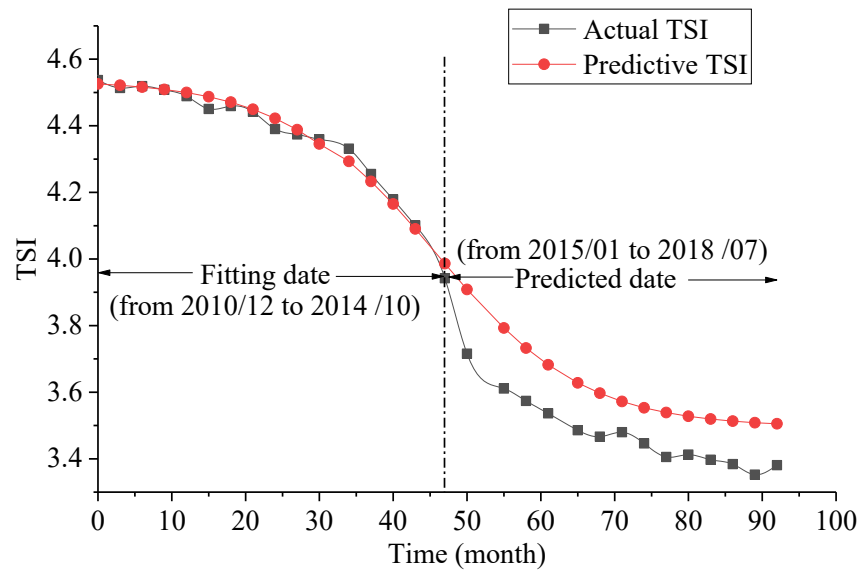

The tunnel entered long-term serviceability degradation after June 2015 (when the TSI decrease rates were smaller than $0.2 /$ year). Therefore, the model for predicting long-term serviceability was used to predict the serviceability of the tunnel after June 2015. Take the TSI value in June 2015 (3.61) as $T S I_{0}$, and input the data during the period from June 2015 to July 2018 into Equation (3) for fitting. The resulting prediction of the serviceability of the tunnel during this period can be expressed as Equation (6):

$T S I=3.61 \times\left[1-e^{-\left(\frac{300.41}{x-4.50}\right)^{0.21286}}\right], \quad(\mathrm{R}=0.989)$

Fig. 10 presents the predicted serviceability degradation curve of the tunnel during this period. The long-term degradation of the tunnel fits with the concave-curve mode of degradation. More specifically, the tunnel experienced rapid degradation during the initial stage of operation, and the degradation slowed down, owing to the controls implemented by the tunnel operator. As predicted by the model, the TSI of the tunnel will decrease to below 3.0 after 26 years of operation (or the serviceability will degrade from the Good level to the Fair level), and will decrease to 2.6 after 100 years of operation (or degrade to the Fair level, though still acceptable) ( $\mathrm{Li}$ et al., 2017). However, the model is needed to be updated in a timely manner with subsequent monitoring data to ensure the longterm validity of it. 
Figure 10 Prediction curve of the long-term decay period

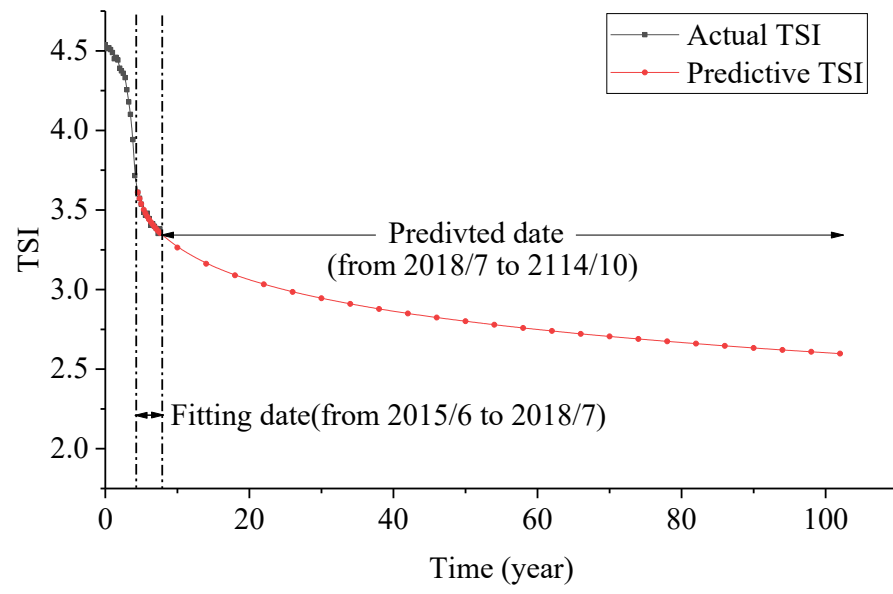

\section{Conclusion}

(1) The serviceability of metro shield tunnels in soft ground generally exhibits a pattern of rapid degradation followed by long-term degradation. If the TSI of a tunnel continuously decreases at rates higher than 0.05 /quarter for a period longer than a year, then the period is defined as a period of rapid degradation.

(2) Based on maintenance characteristics, the rapid serviceability degradation of a tunnel can be categorized into two distinct modes, slowing-down degradation and recoveryand-slowing-down degradation. Both modes of rapid degradation can be well predicted, by using a Sigmoid function.

(3) The long-term serviceability degradation of shield tunnels can be categorized into three distinct modes, concave-curve, Sshaped-curve, and convex-curve modes. All three modes of long-term degradation can be well predicted, by using an inverse exponential function.

(4) The capability of the above models for predicting the serviceability degradation of tunnels was validated, by applying them to a metro shield tunnel in soft ground against eight years of monitoring and maintenance data.

\section{Acknowledgements}

This work is supported by National Key R\&D Program of China (2017YFB1201204) and the Fundamental Research Funds for the Central Universities (2017-2019)

\section{References}

Yuan Y et al. (2012) Assessment service state of tunnel structure. Tunnelling \& Underground Space Technology 27(1): 72-85, 10.1016/j.tust.2011.07.002.

Yuan Y et al. (2013) Predictive maintenance of shield tunnels. Tunnelling \& Underground Space Technology 38(3): 69-86, 10.1016/j.tust.2013.05.004.

Huang $\mathrm{H}$ et al. (2017) Field data-based probabilistic assessment on degradation of deformational performance for shield tunnel in soft clay. Tunnelling \& Underground Space Technology 67: 107-119, 10.1016/j.tust.2017.05.005.

Liu S et al. (2017) Erosion Degradation Mechanism of Shield Tunnel Lining Structure in Corrosive Ion Environment. China Journal of Highway and Transport 30(8): 125-133.

Wei G et al. (2009) Application of Mathematic Models in Prediction of Long-term Surface Settlements above Shielddriven Tunnels. Chinese Journal of Underground Space and Engineering 5(3): $\quad 541-545, \quad 10.3969 /$ j.issn.16730836.2009.03.026.

Li X et al. (2017) Condition assessment of shield tunnel using a new indicator: The tunnel serviceability index. Tunnelling \& Underground Space Technology 67: 98-106, 10.1016/j.tust.2017.05.007.

Wang R and Zhang D. (2013) Mechanism of transverse deformation and assessment index for shield tunnels in soft clay under surface surcharge. Chinese Journal of Geotechnical Engineering 35(6):1092-1101. 